\title{
A NOTE ON CAUCHY'S ROOT TEST
}

\author{
by I. S. MURPHY
}

(Received 25 April, 1975)

Throughout this note we deal with a series $\sum a_{n}$ of positive terms. The following tests for the convergence of this series are well-known.

TEST 1. (Ratio test). Let $\lim a_{n+1} / a_{n}=K$. Then, if $K<1, \sum a_{n}$ converges, while if $K>1, \sum a_{n}$ diverges. diverges.

TEST 2. (Root test). Let $\lim _{n \rightarrow \infty} a_{n}^{1 / n}=K$. Then, if $K<1, \sum a_{n}$ converges, while if $K>1, \sum a_{n}$

It is known that Test 2 is more powerful than Test 1. Theorem 5.3 of Phillips [3] states that if $\lim _{n \rightarrow \infty} a_{n+1} / a_{n}=K$, then $\lim _{n \rightarrow \infty} a_{n}^{1 / n}=K$. This shows that Test 2 is at least as powerful as Test 1 . In the wake of this, consideration of a series like

$$
a+a+a^{2}+a^{2}+a^{3}+a^{3}+\ldots \quad(0<a<1),
$$

to which Test 2 applies while Test 1 does not, shows that Test 2 is strictly more powerful than Test 1 .

A third test is Gauss's test. Like the Ratio test, it scans the ratio of successive terms. It is however much more incisive than the Ratio test. The form of Gauss's test given as Test 3 is Ex.34.1 of Rankin [4]. Other forms occur in Bromwich [1] and Knopp [2].

TEST 3. (Gauss's test). Suppose that there exists $X$ such that for all $n \geqq X$,

$$
a_{n+1} / a_{n}=1-\alpha / n+\varepsilon_{n} .
$$

(i) If $\alpha>1$, and $\varepsilon_{n}=o(1 / n)$, then $\sum a_{n}$ converges.

(ii) If $\alpha<1$, and $\varepsilon_{n}=o(1 / n)$, then $\sum a_{n}$ diverges.

(iii) If $\alpha=1$, and $\varepsilon_{n}=o(1 /(n \log n))$, then $\sum a_{n}$ diverges.

- The purpose of this note is to provide a fourth test. The relationship between Test 4 and Gauss's test is analogous to the relationship between the Root test and the Ratio test.

TEST 4. Suppose there exists $X$ such that for all $n \geqq X$,

$$
a_{n}^{1 /(n \log n)}=1-\alpha / n+\varepsilon_{n} .
$$

(i) If $\alpha>1$, and $\varepsilon_{n}=o(1 / n)$, then $\sum a_{n}$ converges.

(ii) If $\alpha<1$, and $\varepsilon_{n}=o(1 / n)$, then $\sum a_{n}$ diverges.

(iii) If $\alpha=1$, and $\varepsilon_{n}=o(\log \log n /(n \log n))$, then $\sum a_{n}$ diverges.

Proof. Parts (i) and (ii) are similar. We prove part (ii). Suppose that $\alpha<1$ and $\varepsilon_{n}=o(1 / n)$. Then for all $\varepsilon>0$, there exists an integer $N$ such that

$$
a_{n}^{1 /(n \log n)} \geqq 1-(\alpha+\varepsilon) / n \text { for all } n \geqq N .
$$


Choosing $\varepsilon=\frac{1}{2}(1-\alpha)$, we deduce that, for all sufficiently large $n$,

$$
\begin{aligned}
a_{n} & \geqq[1-(\alpha+1) / 2 n]^{n \log n} \\
& =\exp \{n \log n \log [1-(\alpha+1) / 2 n]\} \\
& =\exp \left[-\frac{1}{2}(\alpha+1) \log n+O(\log n / n)\right] \\
& \geqq \frac{1}{2} n^{-\frac{1}{2}(\alpha+1)}
\end{aligned}
$$

So $\sum a_{n}$ diverges by comparison with $\sum n^{-\frac{1}{2}(\alpha+1)}$.

To prove part (iii), suppose that $\alpha=1$ and that $\varepsilon_{n}=o(\log \log n /(n \log n))$. Then, for all $\varepsilon>0$, we deduce that, for all sufficiently large $n$,

$$
\begin{aligned}
a_{n} & \geqq(1-1 / n-\varepsilon \log \log n / n \log n)^{n \log n} \\
& =\exp [n \log n \log (1-1 / n-\varepsilon \log \log n / n \log n)] \\
& =\exp [-\log n-\varepsilon \log \log n+O(\log n / n)] \\
& \geqq \frac{1}{2} n^{-1}(\log n)^{-\varepsilon} .
\end{aligned}
$$

Taking $\varepsilon=1$, we can deduce that $\sum a_{n}$ diverges by comparison.

The following result shows that Test 4 is at least as powerful as Gauss's test.

THEOREM. Consider the following two conditions.

(A) There exists $X$ such that for all $n \geqq X$,

$$
a_{n+1} / a_{n}=1-\alpha / n+\varepsilon_{n}
$$

(B) There exists $Y$ such that for all $n \geqq Y$,

$$
a_{n}^{1 /(n \log n)}=1-\alpha / n+\delta_{n} \text {. }
$$

Then the following results hold.

(i) If $\alpha \neq 1$ and (A) holds with $\varepsilon_{n}=o(1 / n)$, then (B) holds with $\delta_{n}=o(1 / n)$.

(ii) If $\alpha=1$ and (A) holds with $\varepsilon_{n}=o(1 /(n \log n))$, then (B) holds with $\delta_{n}=o(\log \log n /$ $(n \log n))$.

Proof. (i) Suppose (A) holds with $\alpha \neq 1$ and $\varepsilon_{n}=o(1 / n)$. Then for all $\varepsilon>0$, there exists $N$ such that

$$
1-\frac{\alpha+\varepsilon}{n} \leqq \frac{a_{n+1}}{a_{n}} \leqq 1-\frac{\alpha-\varepsilon}{n} \text { for all } n \geqq N .
$$

So for all $n \geqq N$, we have

$$
\log \left(1-\frac{\alpha+\varepsilon}{n}\right) \leqq \log \frac{a_{n+1}}{a_{n}} \leqq \log \left(1-\frac{\alpha-\varepsilon}{n}\right) .
$$

By increasing $N$, if necessary, and by demanding that $\varepsilon$ is sufficiently small, we can ensure 
that, for all $n \geqq N$,

$$
-\left(\frac{\alpha+2 \varepsilon}{n}\right) \leqq \log \frac{a_{n+1}}{a_{n}} \leqq-\left(\frac{\alpha-\varepsilon}{n}\right) .
$$

So, on summation, we find that

Recalling that

$$
-(\alpha+2 \varepsilon) \sum_{r=N}^{n-1} \frac{1}{r} \leqq \sum_{r=N}^{n-1} \log \frac{a_{r+1}}{a_{r}} \leqq-(\alpha-\varepsilon) \sum_{r=N}^{n-1} \frac{1}{r} .
$$

$$
1+\frac{1}{2}+\frac{1}{3}+\ldots+\frac{1}{n}-\log n \rightarrow \gamma \quad \text { as } \quad n \rightarrow \infty,
$$

we can write

$$
K_{1}-(\alpha+2 \varepsilon) \log (n-1) \leqq \log \frac{a_{n}}{a_{N}} \leqq K_{2}-(\alpha-\varepsilon) \log (n-1),
$$

where $K_{1}$ and $K_{2}$ are constants. Hence

$$
K_{3}-(\alpha+2 \varepsilon) \log (n-1) \leqq \log a_{n} \leqq K_{4}-(\alpha-\varepsilon) \log (n-1),
$$

where $K_{3}$ and $K_{4}$ are constants. Dividing by $n \log n$ and noting that $\log (n-1) / \log n=$ $1+O(1 /(n \log n))$, we deduce that there exist constants $K_{5}$ and $K_{6}$ such that for all sufficiently large $n$

$$
\frac{K_{5}}{n \log n}-\frac{\alpha+2 \varepsilon}{n} \leqq \log a_{n}^{1 /(n \log n)} \leqq \frac{K_{6}}{n \log n}-\frac{\alpha-\varepsilon}{n} .
$$

So, for all sufficiently large $n$,

$$
\log a_{n}^{1 /(n \log n)}=-\alpha / n+o(1 / n),
$$

and therefore

$$
a_{n}^{1 /(n \log n)}=1-\alpha / n+o(1 / n)
$$

(ii) If we start from

$$
1-1 / n-\varepsilon /(n \log n) \leqq \frac{a_{n+1}}{a_{n}} \leqq 1-1 / n+\varepsilon /(n \log n)
$$

for all $n \geqq N$, the proof is similar to the proof of (i) except that in this case we are forced to consider not only $\sum_{r=N}^{n-1} 1 / r$ but also $\sum_{r=N}^{n-1} 1 /(r \log r)$. The latter can be dealt with by using the fact that

$$
\frac{1}{2 \log 2}+\frac{1}{3 \log 3}+\ldots+\frac{1}{n \log n}-\log \log n
$$

tends to a finite limit as $n \rightarrow \infty$.

COROLlaRY. When Gauss's test works, so also does Test 4. 
Remarks. 1. That Test 4 is strictly more powerful than Gauss's test can be seen from the series $\sum a_{n}$, where $a_{2 n-1}=a_{2 n}=[1-(3 / 2 n)]^{2 n \log 2 n}$ for all $n \geqq 1$. Here Gauss's test is inapplicable since $a_{2 n} / a_{2 n-1}=1$, for all $n \geqq 1$. However, $a_{n}^{1 /(n \log n)}=1-(3 / n)+o(1 / n)$ for large values of $n$, so that the series converges by Test 4 .

2. In practice, it is found that the Ratio test is more useful than the Root test, despite being less powerful. So also is it with Gauss's test and Test 4.

\section{REFERENCES}

1. T. J. I'A. Bromwich, Infinite series (Macmillan, 1908).

2. K. Knopp, Theory and application of infinite series (Blackie, 1928).

3. E. G. Phillips, $A$ course of analysis (Cambridge, 1962).

4. R. A. Rankin, An introduction to mathematical analysis (Pergamon, 1963).

UNIVERSITY OF GLASGOW

SCOTLAND

\section{CORRIGENDUM}

to the paper

\section{ABELIAN ERGODIC THEOREMS FOR VECTOR-VALUED FUNCTIONS $\dagger$}

by P. E. KOPP

The definition of $\Omega_{f, e}$ on p. 57 should read

$$
\Omega_{f, a}=\bigcup_{0<\rho<1}\left\{w \in \Omega:(1-\rho)\left\|\left(R_{\rho} f\right)(w)\right\|_{X}>a\right\} .
$$

† Vol. 16 (1975), 57-60. 